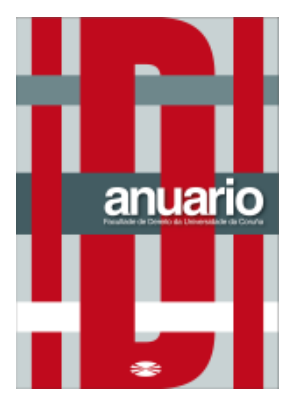

Anuario da Facultade de Dereito da Universidade da Coruña

Vol. 24 (2020), pp. 231-240

ISSNe: 2530-6324 || ISSN: 1138-039X

DOI: https://doi.org/10.17979/afdudc.2020.24.0.7498

\title{
COLABORACIÓN INTERUNIVERSITARIA INTERNACIONAL EN UN CONTEXTO INTERDISCIPLINAR
}

\section{INTERNATIONAL INTERUNIVERSITY COLLABORATION IN AN INTERDISCIPLINARY CONTEXT}

\begin{abstract}
ASUNCIÓN LÓPEZ ARRANZ (a.larranz@udc.es)
Profesora Contratada Doctora

Departamento de Derecho Público

Área Derecho del Trabajo de la Seguridad Social

Economic Development and Social Sustainability Research Group EDaSS

Grupo de Innovación Docente TI4HE - Tecahing Innovation for Higher Education

Universidade da Coruña

https://orcid.org/0000-0002-9058-9044

XOSE PICATOSTE (j.pnovo@udc.es)

Departamento de Economía

Área de Fundamentos del Análisis Económico

Economic Development and Social Sustainability Research Group EDaSS

Grupo de Innovación Docente TI4HE - Tecahing Innovation for Higher Education

Universidadade da Coruña

Miembro Colaborador del Gupo de investigación SET-LASE - Socioeonomía del Trabajo

(Universidad Autónoma de Madrid)

https://orcid.org/0000-0002-5761-771X
\end{abstract}

Recibido: $18 / 04 / 2020$

Aceptado: 23/05/2020

Resumen: La importancia de la colaboración en el ámbito de la docencia superior es cada vez más reconocida. Los mejores resultados académicos surgen como consecuencia del aumento del interés de los estudiantes, estimulados por la diversidad de contenidos y enfoques. En este trabajo se presenta una experiencia multidisciplinar e internacional de colaboración en la docencia superior -un curso de postgrado-, realizado durante los años 
académicos 2016/2017, 2017/2018, 2018/2019 y 2019/2020. El objetivo principal de esta presentación es poner el foco en las ventajas de la docencia interdisciplinar en general, y de las sinergias entre los campos jurídico y económico. La metodología docente ha sido mixta: presencial y a distancia.

Palabras clave. Docencia interdisciplinar, Postgrado, Mercado de trabajo, Ciencias sociales, Derecho y economía.

Abstract: The importance of collaboration in the field of higher education is increasingly recognized. The best academic results emerge as a consequence of the increased interest of students, stimulated by the diversity of content and approaches. This work presents a teaching experience for a postgraduate course, carried out during several courses 20162017, 2017-2018 and 2019-2020 of a multidisciplinary and international experience collaboration in higher teaching. The main objective of this presentation is to focus on the advantages of interdisciplinary teaching in general, and the synergies between the legal and economic fields. The teaching methodology has been mixed: face-to-face and distance.

Keywords: Interdisciplinary teaching, Postgraduate, Labour market, Social sciences, Law and economics.

\section{Sumario: I. ANTECEDENTES. II. OBJETIVOS Y METODOLOGÍA. III. FUNDAMENTACIÓN DE LA DOCENCIA COLABORATIVA. IV. RESULTADOS. V. CONCLUSIONES. BIBLIOGRAFÍA}

\section{ANTECEDENTES}

Uno de los principales retos de la docencia es la motivación de los alumnos, como primer paso para lograr un aprendizaje consciente, productivo, realista que les permita conseguir competencias y objetivos ${ }^{1}$. Lograr la motivación en el aula y alcanzar un aprendizaje proactivo, es el deseo de todos los docentes y uno de los más firmes propósitos al inicio de cada curso escolar. La realidad demuestra que un importante porcentaje de alumnos carece de motivación en el aula ${ }^{2}$, por ese motivo la experiencia que aquí se relata se basa en un planteamiento inicialmente aplicado a una docencia internacional con alumnos de otra universidad, extranjera, y otro idioma vehicular, conjuntamente con una metodología multidisciplinar que permitiese hacer una comparativa entre los alumnos de

1 Véase YÁNIZ, C., Y VILLARDÓN, L. "Planificar desde competencias para promover el aprendizaje”, Universidad de Deusto, ( Vol. 12), 2008, p. 25.

2 Véase CORCHUELO RODRIGUEZ, C. A. "Gamificación en educación superior: experiencia innovadora para motivar estudiantes y dinamizar contenidos en el aula”, Edutec. Revista Electrónica de Tecnología Educativa (63), 2018, pp. 29-41. En el mismo sentido, véase MONTICO, S., "La motivación en el aula universitaria: ¿una necesidad pedagógica?”, Ciencia, docencia y tecnología, 15(29), 2004, pp. $105-112$. 
las universidades de ambos países participantes (España y Portugal), en concreto, se ha centrado el análisis en el estudiantado de la Universidad de A Coruña y de la Universidad Católica de Oporto. Esta experiencia comenzó en el curso 2016-2017 y ha continuado hasta la actualidad, en el curso 2019-2020. En todo el proceso se han utilizado diversas herramientas de aprendizaje con Tecnologías dela Información y la Comunicación (TICs). En esta propuesta de trabajo se detalla una experiencia docente y se describen los resultados comparativos entre los alumnos de ambas universidades.

\section{OBJETIVOS Y METODOLOGÍA}

Se planteó, desde el primer momento de su organización, un complejo y detallado diseño de del contenido del programa a impartir. Simultáneamente se estableció un sistema de flujo de información continua, con el objeto de establecer un sistema de coordinación estable, permanente y dinámico. En primer lugar, se planteó por los equipos docentes fue la necesidad de coordinación entre los mismos, ya que, por un lado, se encontraban en distintas universidades y por otro, en distintas áreas de conocimiento. La segunda cuestión fue la preparación de un programa dirigido a alumnos de distintas titulaciones y de distintos países. En cuanto a la primera se acordó que los profesores portugueses se ocuparan de las cuestiones administrativas mientras que el equipo docente español se ocuparía de la docencia. Y en cuanto a la elaboración del programa esta se haría de forma conjunta, tras una propuesta presentada por el equipo español, el equipo portugués manifestó su total acuerdo. También los diferentes equipos de profesores universitarios debieron tomar una decisión respecto a los objetivos, contenidos, metodologías y formas de evaluación que garantizarían un enfoque interdisciplinar y que, además, fueran significativos para la formación docente. Una representación sintética y esquemática de este proceso se refleja en la Figura 1.

Figura 1. Resumen esquema implementación docencia

\begin{tabular}{|c|}
\hline Contenido de la programación \\
\hline Coordinación interuniversitaria \\
\hline Coodinación interdisciplinar \\
\hline Diseño de materiales presenciales y online \\
\hline Distirbución/Impartición docencia \\
\hline Coordinación de la Evaluación y la calificación \\
\hline
\end{tabular}

Fuente: elaboración propia

Los equipos docentes se plantearon el desafío del mencionado trabajo como una gran oportunidad que los llevo a reformular tanto objetivos como estrategias ya que en este curso hubo que concordar materias tan diferentes y al mismo tiempo tan complementarias como son la economía y el derecho. Por otra parte, también se llevó a cabo un cambio en la 
modalidad de docencia, dotando, por tanto, al curso de importantes innovaciones, las cuales vinieron de la mano de las TICs y del aprendizaje colaborativo ${ }^{3}$.

El objetivo principal de esta experiencia fue poner el foco en las ventajas de la docencia interdisciplinar en general, y de las sinergias entre los campos jurídico y económico. Simultáneamente, se conjugaron las modalidades de docencia online y presencial $^{4}$. El equipo docente se planteó que, si nuestra sociedad tecnológica ha obtenido sus mejores resultados a través del trabajo interdisciplinar, y que, si esto es lo que demanda la sociedad, en materia de desarrollo científico e innovación, el abordaje interdisciplinario de los contenidos académicos es también una necesidad, de lo contrario los alumnos no estarán preparados para desenvolverse en un mundo que es cada vez más complejo e interconectado ${ }^{5}$. Así, dado que las dos ciencias cumplen con una importante demanda de la sociedad, la formación conjunta de economía y derecho que se llevó a cabo en este curso fue considerada de una gran importancia ${ }^{6}$. La docencia Universitaria en este curso ha cumplido con las ideas básicas que están impresas en el ADN de la universidad como son, lugar para conocer, investigar, preguntar, producir, socializar y vivenciar en definitiva una formación de gran calidad de la mano de la interdisciplinaridad ${ }^{7}$. En definitiva, estar a la vanguardia de la sociedad, aportando un conocimiento sólido y fundamentado para el avance en un mundo globalizado, donde ni los espacios geográficos ni los nichos de cada disciplina se pueden desarrollar aisladamente8. Por otra parte, como valor adicional, se contextualiza esta docencia en un entorno interuniversitario en el que se ha comprometido dos países, España y Portugal. La colaboración en la formación universitaria entre los dos países ha sido y sigue siendo muy estrecha, al haber creado redes de contacto y colaboración científica entre profesores y alumnos. Además, desde la perspectiva de la Universidad de la Coruña y la Universidad Católica de Oporto, esta colaboración ha sido siempre de gran importancia por los lazos culturales que unen a ambos pueblos. Adicionalmente, dentro de los objetivos estratégicos de ambas universidades se encuentra la internacionalización como medida de impuso para una docencia de calidad.

La metodología docente ha sido mixta: presencial y a distancia, durante dos meses, distribuida en 1-2 días por semana. Los docentes han sido todos españoles y los alumnos

${ }^{3}$ Véase JORRIN, I., VEGA, G. y GÓMEZ, E. "El papel facilitador de las Tic en un proceso de aprendizaje colaborativo”, Revista Latinoamericana de Tecnología educativa, nº 3, 2004, págs. 260-267.

${ }^{4}$ Véase NOVO-CORTI, I., VARELA-CANDAMIO, L. y RAMIL-DÍAZ, M., "E-learning and face to face mixed methodology: Evaluating effectiveness of e-learning and perceived satisfaction for a microeconomic course using the Moodle platform”, Computers in Human Behavior, 29(2), 2013, pp. 410415.

${ }^{5}$ Véase BURGUERA, J. L., PÉREZ-HERRERO, M. D. H. y VIRGÓS-SÁNCHEZ, M., “Educational coaching as a methodological strategy for the career development in 4th grade of secondary education 'guideyou' a multidisciplinary approach”, Proceedings of the Fourth International Conference on Technological Ecosystems for Enhancing Multiculturality, 2016, pp. 205-209.

${ }^{6}$ Véase ARTHUR, M. B., (2008), "Examining contemporary careers: A call for interdisciplinary inquiry”, Human relations, 61(2), pp. 163-186.

${ }^{7}$ Véase CÁRDENAS PÉREZ, R. CASTRO ORELLANA, A. M. y SOTO BUSTAMANTE, "El desafío de la interdisciplinariedad en la formación de docentes”, Revista electrónica diálogos educativos, n 1, 2001, p. 19.

${ }^{8}$ Véase STIGLITZ, J. E. y GREENWALD, B. C., (2016), "La creación de una sociedad del aprendizaje: Una nueva aproximación al crecimiento, el desarrollo y el progreso social”, La Esfera de los Libros, pp. 125300. 
todos portugueses. El idioma de impartición de la docencia ha sido fundamentalmente inglés, aunque se ha combinado con el portugués y el gallego. El diseño metodológico de la docencia ha recaído completamente en los profesores españoles, mientras que los profesores portugueses han participado únicamente en las gestiones organizativas.

En definitiva, el curso vinculado al ámbito jurídico-social, como experiencia en la Universidad Católica de Oporto ampliado las perspectivas docentes para alumnos y profesores. Para los primeros, el curso pretendió la inmersión del aprendizaje de forma global del Derecho español y portugués, así como de la economía española y portuguesa. Esto se enmarca en un contexto globalizador y de interacción cultural ${ }^{9}$. Presentando factores contextuales y personales ${ }^{10}$. Por otro lado, la experiencia también supuso un impulso en la calidad de la carrera profesional de los profesores, tanto en el ámbito docente como investigador ${ }^{11}$.

\section{FUNDAMENTACIÓN DE LA DOCENCIA COLABORATIVA}

El intento por analizar diferencias y puntos en común, así como la de fortalecer la preparación de los profesores a través de la colaboración interdisciplinar e internacional llevó a los participantes a proponer este curso pivotando sobre los puntos en común y la complementariedad de las materias jurídicas y de economía y empresa. Ambas regulan normas de convivencia de las personas que conforman la sociedad. Siendo las relaciones sociales, aquellas que se manifiestan en la búsqueda por satisfacer necesidades el aspecto esencial para la economía, mientras que el derecho, es garantista de lo que busca la economía. Por ello, en definitivo el objetivo en el estudio de estas dos ciencias es el comportamiento humano, base del planteamiento de este trabajo ${ }^{12}$.

La docencia planteada se centró en el mercado de trabajo bajo la perspectiva jurídico-económica. Las sesiones presenciales se dividieron a partes iguales entre las disciplinas de derecho y de economía, de forma intercalada. Se parte de un esquema docente de base telemática, con aspectos teóricos y prácticos, que los alumnos conocen desde el inicio. En las primeras clases de la materia jurídico-económica se repasan los conocimientos básicos en los contenidos de derecho positivo laboral y economía, para lo cual se prepara un cuestionario a través de Kahoot, que deben contestar, tras lo cual se explican cada una de las preguntas y se va desgranado la materia teórica. De esta manera se desarrolla el programa teórico, se explica el tema, se realiza un cuestionario teórico y posteriormente se aclaran las posibles dudas. En cuanto a la materia Práctica se realiza del mismo modo. En el caso de las sesiones jurídico laborales se ha comenzado con una

\footnotetext{
${ }^{9}$ En este sentido, cabe resaltar que, en el momento inicial de esta colaboración, uno de los coautores de este trabajo se encontraba en la Universidad Autónoma de Madrid, la cual, por tanto, ha participado también en esta experiencia.

10 Véase ARTUNDUAGA, M., Variables que influyen en el rendimiento académico en la Universidad (Tesis Doctoral inédita), Universidad Complutense de Madrid, Madrid, 2008, pp. 55-60.

11 Véase LOREDO ENRÍQUEZ, J., ROMERO LARA, R. e INDA ICAZA, P., “Comprensión de la práctica y la evaluación docente en el posgrado a partir de la percepción de los profesores”, Revista electrónica de investigación educativa, 2008, vol. 10, no SPE, pp. 1-16.

12 Véase ROBBINS L., “Ensayo sobre la naturaleza y significancia de la Ciencia Económica”, Londres1932, pp. 50-100 (http://www.eumed.net/cursecon/textos/robbins/index.htm).
} 
presentación de la situación del marco legal en España en relación a los Derechos de carácter laboral recogidos en la Constitución Española de 1978 y a la normativa laboral como es el Estatuto de los Trabajadores de $2015^{13}$. En materia de derechos sociales la Constitución Española de 1978 constitucionalizó una serie de derechos, principios y directrices, de nuestro sistema de relaciones laborales. Fundamentándose en que las relaciones de trabajo son relaciones de poder, con un gran impacto social y en el hecho de que el mercado en el que se desarrollan tienen un elevadísimo impacto económico y social $^{14}$. La finalidad era la de analizar el estado de reforma permanente de nuestro derecho del trabajo dividido en cuatro grandes etapas que van desde 1980 a $2010-11^{15}$, a la que debemos añadir una fase previa o de "gestión de la crisis", particularmente intensa, que culminó con el Estatuto de 1980 y una fase final, protagonizada por la reforma de $2012^{16}$. Si este fue el marco español en Portugal se analizaron de forma paralela tanto los derechos laborales recogidos en su Constitución de 1976 como en el Código do Traballo portugués modificado en 2009, así como las reformas de carácter laboral que en los años 2010 y 2011 sufrió la legislación portuguesa ${ }^{17}$. A continuación se presentaron las principales magnitudes macroeconómicas sobre economía general y mercado de trabajo ${ }^{18}$, centradas en la evolución de la producción y el empleo y en el funcionamiento de una economía en su conjunto, especialmente enfocada hacia el estudio de fiscales y laborales de los mercados de trabajo ${ }^{19}$. Al igual que en las presentaciones relativas al marco normativo, se han hecho referencias comparativas a la situación y evolución de la situación en España y Portugal. Realizadas las sesiones descritas, se presentaron trabajos de los alumnos, de forma presencial e interactiva. Así, el caso (que describe una situación profesional en la vida real) ha de ser comprendido, valorado y resuelto a través de un proceso de discusión, para llegar a una decisión razonada. El proceso es siempre coordinado por los profesores, tanto de economía como derecho.

Los trabajos se han realizado en Portugal con asistencia on-line desde España, por parte de los profesores españoles. Se ha facilitado material en versión digital, así como bases de datos oficiales. La elección de las tareas propuestas se centraban en fomentar el trabajo en equipo, y por otro para profundizar en los temas programados, enfocados en el

13 Véase “La Legislación Laboral Básica”, Civitas, Aranzadi, Tecnos, 2016.

14 Véase GARCÍA ABELLAN J., Derecho Sindical, Universidad de Murcia, 1984, pp. 50-100, y OJEDA AVILES, A., Derecho Sindical, ed. Tecnos, Madrid, 2003, pp. 325-365. SALA FRANCO, T. y ALBIOL MONTESINOS, I., Derecho Sindical, ed. Tirant Lo Blanch, 2003, pp. 125-145. Y RODRÍGUEZPIÑERO, M. y OTROS, Comentarios a la Ley de Libertad sindical, Tecnos, 1986, pp. 1-100.

15 Véase MARTÍNEZ GIRÓN, J., ARUFE VARELA, A. y CARRIL VÁQUEZ, X.M., Derecho del Trabajo, Netbiblo, 2006, pp. 125-168.

${ }^{16}$ Véase a CRUZ VILLALÓN, J., “Hacia un nuevo modelo laboral en España”, Revista de la Facultad de Derecho PUCP, ${ }^{\circ}$ 68, Sevilla, 2012, pp. 132-156.

${ }^{17}$ Véase RIBEIRO MENDES, F., “Segurança Social o futuro hipotecado”, Ensaios da Fundaçao, Lisboa, 2011, pp. 40-41.

18 Véase GINDAL, M., “Los días que vivimos peligrosamente”, Planeta, 2012, pp. 225-300.

19 Véase PICATOSTE, X., La devaluación interna en el contexto de la actual economía española, Trabajo Fin de Grado. Universidad de A Coruña, 2014, pp. 50-100, y PICATOSTE, J., RUESGA-BENITO, S. y GONZÁLEZ-LAXE, F., "Economic sustainability and possibilities of action for the states, in the case of monetary integration: some notes for reflection”, Progress in Industrial Ecology, an International Journal, 10(1), 2016, 16-33. 
desarrollo de diferentes competencias en los alumnos ${ }^{20}$. El trabajo en equipo permitió desarrollar la capacidad de trabajo, el compromiso, la responsabilidad y resultados de calidad en los alumnos. Individualmente, se logró aumentar las habilidades en la utilización de fuentes de información científica, tanto de ámbito jurídico laboral como económico y organizacional, así como de interpretación y comunicación. Asimismo, se trabajaron competencias como la gestión del tiempo, las habilidades comunicativas, la claridad y organización de las exposiciones, la capacidad de debate y la creatividad ${ }^{21}$.

Desde el inicio, al alumno se le proporciono un programa detallado con los diferentes módulos del curso, consensuados entre los profesores españoles y portugueses. La evaluación se ha realizado tomando en cuenta el trabajo en el aula, y se han utilizado el Kahoot y evaluación de trabajos prácticos en grupos e individuales ${ }^{22}$.

\section{RESULTADOS}

Los resultados de la docencia no muestran diferencias entre alumnos de los dos países y universidades, sí se ha comprobado una mayor implicación con respecto a los estudiantes de otros cursos de postgrado convencionales. En síntesis, se podrían resumir como sigue:

Por parte del alumno:

> Más implicación del alumno en el aprendizaje responsable y colaborativo, dado que, en el caso del aprendizaje en grupo, el beneficio o perjuicio de uno de su miembro es muy relevante para todo el grupo.

$>$ Mayor participación en el aula.

$>$ Aumento de la motivación y adquisición de competencias, así como mejores resultados de aprendizaje.

$>$ Mejora en los resultados académicos.

Por parte del profesor

> Hemos podido comprobar como el comportamiento del alumnado de máster es igual en la Universidad Católica que en la Universidad de A Coruña, que han adquirido las mismas competencias como la docencia impartida, han mostrado el mismo interés por participar en la clase y con los trabajos y han recibido al profesorado de otra universidad como si fuera el suyo propio.

\section{CONCLUSIONES}

${ }^{20}$ Véase MESIA DE LA CERDA, J.A. y FERNÁNDEZ VICENTE, E., Innovación educativa para la educación superior: hacia el proceso de convergencia, Dikynson, 2009, pp. 574-579.

${ }^{21}$ DE PABLOS PONS, J., "Universidad y sociedad del conocimiento, las competencias informacionales y digitales”, Revista de Universidad y Sociedad del Conocimiento, nº 7, 2010, pp. 7-12.

${ }^{22}$ Véase JIMÉNEZ VALVERDE, G., "Obtención de notas individuales a partir de una nota de grupo mediante una evaluación cooperativa”, Revista Iberoamericana de Educación, Vol. 38, núm. 5, 2006, pp. 210. 
La integración de las TICs como herramienta ha facilitado mucho la comunicación, la organización previa de las clases y la motivación de alumno, de la misma manera que ocurre con los alumnos españoles. Se ha podido comprobar que el aprendizaje fue significativo, real y continuo, ya que las preguntas formuladas en los diversos cuestionarios se han ido entrelazando con todo el programa de las materias, por lo que a la finalización de las clases el alumno había obtenido un aprendizaje claro y solido del programa, habiendo adquirido las competencias propuestas en el mismo. Todo ello ha quedo demostrado por unos resultados académicos importantes de las materias impartidas en el curso con un índice de aprobados superior al 90\%.

Esta experiencia es valiosa en sí misma, pero, además, se torna más interesante, si cabe, en contextos de movilidad y agrupamiento restringido, tal como ha ocurrido con la pandemia originada por la COVID-19. Por tanto, se podría considerar esta aportación como un experimento piloto y de referencia.

\section{BIBLIOGRAFÍA}

ARTHUR, M. B., “Examining contemporary careers: A call for interdisciplinary inquiry”, Human relations, 61(2), 2008.

ARTUNDUAGA, M., Variables que influyen en el rendimiento académico en la Universidad (Tesis Doctoral inédita), Universidad Complutense de Madrid, 2008.

BURGUERA, J. L., PÉREZ-HERRERO, M. D. H. y VIRGÓS-SÁNCHEZ, M., "Educational coaching as a methodological strategy for the career development in 4th grade of secondary education. 'guide-you' a multidisciplinary approach”, en Proceedings of the Fourth International Conference on Technological Ecosystems for Enhancing Multiculturality, 2016.

CÁRDENAS PÉREZ ANA, CASTRO ORELLANA RODRIGO, SOTO BUSTAMANTE ANA MARÍA, "El desafió de la interdisciplinariedad en la formación de docentes", Revista Electrónica diálogos educativos, nº 1, 2001.

CORCHUELO RODRIGUEZ, C. A., "Gamificación en educación superior: experiencia innovadora para motivar estudiantes y dinamizar contenidos en el aula”, Edutec. Revista Electrónica de Tecnología Educativa, (63), 2018.

CRUZ VILLALÓN, J., "Hacia un nuevo modelo laboral en España”, Revista de la Facultad de Derecho PUCP, $\mathrm{n}^{\circ}$ 68, Sevilla, 2012.

DE PABLOS PONS, J., "Universidad y sociedad del conocimiento. Las competencias. Universidad y sociedad del conocimiento”, RUSC, Universities and Knowledge Society Journal, 7(2), 2010. 
GARCÍA ABELLAN, J., Curso de Derecho Sindical, Universidad de Murcia, 1984.

GUINDAL, M., Los días que vivimos peligrosamente, Colección Booket, Editorial Planeta S.A, 2012.

JIMÉNEZ VALVERDE, G., "Obtención de notas individuales a partir de una nota de grupo mediante una evaluación cooperativa”, Revista Iberoamericana de Educación, Vol. 38, 2006, núm. 5.

JORRIN, I, VEGA G Y GÓMEZ. E., "El papel facilitador de las Tic en un proceso de aprendizaje colaborativo", Revista Latinoamericana de Tecnología educativa, $\mathrm{n}^{\circ} 3$, RELATEC, 2004.

LEGISLACIÓN LABORAL BÁSICA. Civitas, Aranzadi y Tecnos, 2016.

LOREDO ENRÍQUEZ, J, ROMERO LARA, R, INDA ICAZA, P., "Comprensión de la Práctica y la evaluación docente en el posgrado a partir de la percepción de los profesores”, Revista electrónica de investigación educativa, Número especial, 2008.

MARTÍNEZ GIRÓN, J. ARUfE VARELA, A. CARRIL VÁQUEZ, X. M., Derecho del Trabajo, ed. Netbiblo. 2006, A Coruña.

MESIA DE LA CERDA, J.A. Y FERNÁNDEZ VICENTE, E., Innovación educativa para la educación superior: hacia el proceso de convergencia, Dikynson (Madrid, 2009).

MONTICO, S., La motivación en el aula universitaria: ¿una necesidad pedagógica? Ciencia, docencia y tecnología, 15(29), 2004.

MURILLO, P., Nuevas formas de trabajar en la clase: metodologías activas y colaborativas. En Abello l. (Ed). El desarrollo de competencias docentes en el profesorado, Ministerio de Educación. Madrid, 2007.

NOVO-CORTI, I., VARELA-CANDAMIO, L. Y RAMIL-DÍAZ, M., (2013). “E-learning and face to face mixed methodology: Evaluating effectiveness of e-learning and perceived satisfaction for a microeconomic course using the Moodle platform”. Computers in Human Behavior, 29(2).

OJEDA AVILÉS, A., Derecho Sindical, ed. Tecnos, Madrid, 2003.

PICATOSTE, X., La devaluación interna en el contexto de la actual economía española, Trabajo Fin de Grado, Universidad de A Coruña, 2014.

PICATOSTE, J., RUESGA-BENITO, S. Y GONZÁLEZ-LAXE, F., "Economic sustainability and possibilities of action for the states, in the case of monetary integration: some notes for reflection”, Progress in Industrial Ecology, an International Journal, 10(1) 2016. 
RIBEIRO MENDES, F., Segurança Social o futuro hipotecado, Ensaios da Fundaçao, Lisboa, 2011.

ROBBINS, L., Ensayo sobre la naturaleza y significancia de la Ciencia Económica, (http://www.eumed.net/cursecon/textos/robbins/index.htm), Londres, 1932.

RODRÍGUEZ PIÑEIRO, M., Comentarios a la Ley de Libertad sindical: ley orgánica 11/1985, de 2 de agosto de libertad sindical, Tecnos, Madrid, 1986.

SALA FRANCO, T. Y ALBIOL MONTESINOS, I. Derecho Sindical, Tirant lo Blanch, Valencia, 2003.

STIGLITZ, J. E. Y GREENWALD, B. C., La creación de una sociedad del aprendizaje: Una nueva aproximación al crecimiento, el desarrollo y el progreso social, La Esfera de los Libros, Madrid, 2016.

YÁNIZ, C. y VILLARDÓN, L., Planificar desde competencias para promover el aprendizaje (Vol. 12), Universidad de Deusto, 2008. 